\title{
A Fast Algorithm to Track Changes of Direction of a Person Using Magnetometers
}

\author{
A. Fleury, Student Member, IEEE, N. Noury, Senior Member, IEEE, N. Vuillerme
}

\begin{abstract}
Technological advances in signal processing and in circuits integration offer numerous perspectives in telemedicine and telemonitoring. Considering the increase of life expectancy, accurate and reliable assessement of modification and/or deterioration in the health status of a person is needed. One possible indicator is the "activity index" of a person. To compute such an index, previous studies have used accelerometers. Although these sensors are appropriate for the detection of postural transitions (e.g. Sit To Stand and Stand To Sit), they do not allow to detect changes of direction of a walking individual insofar as such activity occurs in a constant gravitation field. Within this context, the purpose of the present work is to investigate whether magnetometers can be used to monitor the changes of direction of a person.
\end{abstract}

Index Terms-Magnetometers, embedded system, yaw angle, quaternion, clustering

\section{INTRODUCTION}

$\mathbf{P}$ ERCENTAGE of population over 65 is increasing and this trend will accelerate in a near future. To reduce the time spent in hospital and to allow older adults to stay at home, smart sensors and smart house [1] can be used not only to monitor the activity, but also to detect abnormal situation in the daily life of the person. Most of these systems integrate accelerometers to detect fall [2] or to classify postural transitions (e.g. Sit To Stand and Stand To Sit) [3]. Data relative to postural transitions of a person can further be used in a data fusion engine to detect and charaterize daily activity of the person and to compute his/her "activity index". At this point, however, accelerometers, by definition, do not allow to detect changes of direction of a walking individual insofar as such activity occurs in a constant gravitation field. Other sensors are thus needed. Interestingly, [4], [5] used a central consisting of accelerometers, magnetometers and gyroscopes to track movements of a limb. In these studies, however, magnetometers are used to compensate the integration drift of the gyroscopes, only. Within this context, the purpose of the present work is to investigate whether magnetometers can be used to monitor the changes of direction of a person.

\section{MATERIALS AND METHODS}

\section{A. Central of sensors - Delivered signals}

As illustrated in Fig. 1, the central of sensors comprises 1 one-axis magnetometer (HMC1021Z, Honeywell) and 1 two-

Anthony Fleury, Norbert Noury, Nicolas Vuillerme are with the Laboratoire TIMC-IMAG, UMR CNRS/UJF 5525, équipe AFIRM. Faculté de Médecine de Grenoble, bâtiment Jean Roget, 38706 La Tronche, France (e-mail: \{Anthony.Fleury,Norbert.Noury,Nicolas.Vuillerme\} @imag.fr).

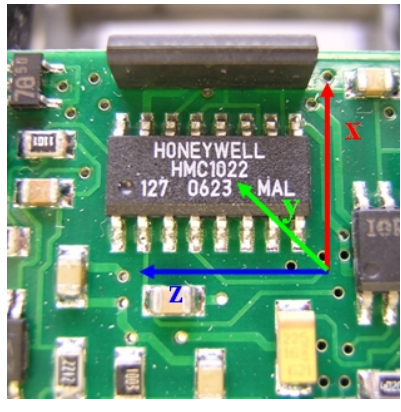

Fig. 1. 3D axis set on the circuit board

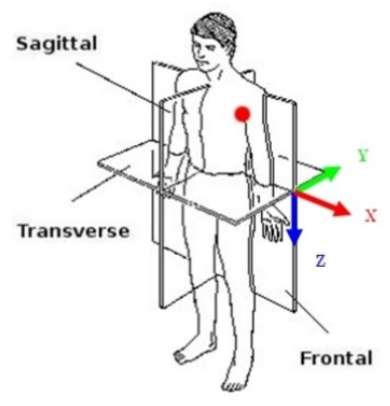

Fig. 2. 3D axis set on the subject (the red point designates location of the sensors) axis one (HMC1022, Honeywell). This central, maintained immobile on the subject's body (Fig. 2), creates a tridimensional direct orthogonal basis, and allows the collection of three distinct signals for $\mathbf{x}, \mathbf{y}$ and $\mathbf{z}$ axes, respectively. An example of collected data from these two magnetometers is given in Fig. 3-A.

\section{B. Signal processing}

To reduce the number of computations, and thus to decrease the calculus consumption of the algorithm, raw signals computed from magnetometers along $\mathbf{x}, \mathbf{y}$ and $\mathbf{z}$ axes are first pre-processed. On the whole, the filters used for both walking and non activity time period detections, are applied on a two seconds window, allowing the use of a relatively rapid algorithm that can be executed in real time.

1) Walk filtering: Considering walking as a periodic signal in the bandwidth $[0.5 \mathrm{~Hz} ; 2 \mathrm{~Hz}]$, the first process is a threeorder bandstop digital filter aimed at reducing the unwanted variations in the magnetometers signal. Note that the signal is duplicated and inverted before and after (mirror effect) to remove discontinuities and hence to prevent edge effects. The second process is a low-pass filter with a $4 \mathrm{~Hz}$ cutoff frequency to remove the high frequency noise. Fig. 3-B and Fig. 4-B. illustrate filtered signals computed from data collected during $250 s$ and $40 s$, respectively. The $40 s$ window, illstrated in Fig. 4-B, corresponds to a temporal frame during which two $180^{\circ}$ "rotations" and a walking sequence were executed.

2) Detection of non-activity periods of time: Since numerous temporal frames contains very few movements and thus do not present any interest, we use a filter which computes 

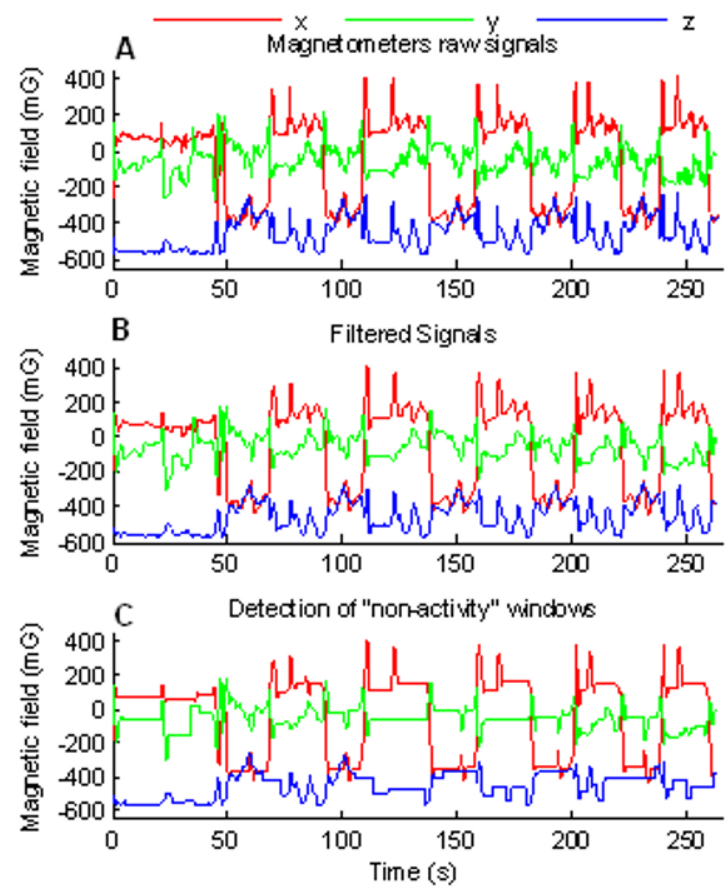

Fig. 3. Successive steps of the filtering process shown on the signal of an experimentation in which the subject walked, sat, stand and made different changes of direction

the running standard deviation of the signal. The standard deviation is defined by the following expression:

$s_{n}=\sum_{i=1}^{n} \frac{\left(x_{i}-\overline{x_{n}}\right)^{2}}{n}=\frac{1}{n}\left(\sum_{i=1}^{n} x_{i}^{2}-2 \cdot \overline{x_{n}} \cdot \sum_{i=1}^{n} x_{i}\right)+{\overline{x_{n}}}^{2}$

where:

$$
\overline{x_{n}}=\frac{1}{n} \cdot \sum_{i=1}^{n} x_{i}=\frac{n-1}{n} \cdot \overline{x_{n-1}}+\frac{1}{n} \cdot x_{n}
$$

This procedure allows to reduce the number of windows to be analyzed, and hence the calculus consumption of the algorithm.

On the one hand, to maintain the continuity of information from one sampling window to another and to avoid saving all collected samples, the following seven floating point parameters have to be computed: (1) the number of samples processed (1 variable), (2) the sum of the samples (1 parameter per axis $=3$ parameters), (3) the sum of the square of the samples ( 1 parameter per axis $=3$ parameters).

On the other hand, to improve the effectiveness of this filter, before collecting magnetometers data, we initialize these seven variables in a temporal frame during which a half-turn movement is executed. This procedure allows the first windows to be defined as "activity windows" only if they really are.

The standard deviation of each window is compared to the running standard deviation. There are two possibilities : (1)

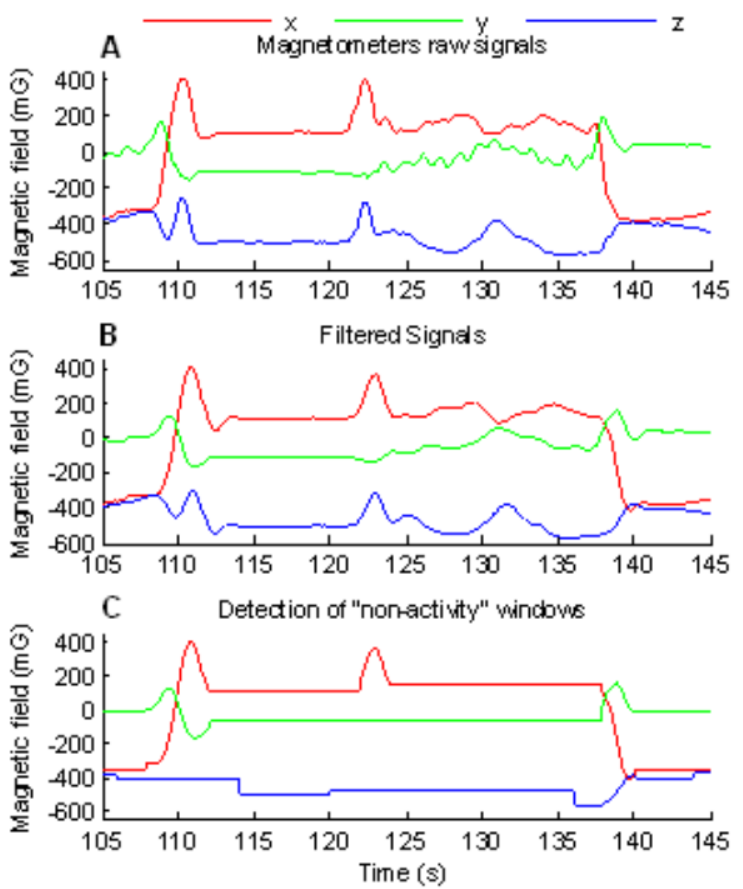

Fig. 4. Successive steps of the filtering process shown on a significant part of the signal of Fig. 3 in which the subject made a $180^{\circ}$ rotation, walked and made a second $180^{\circ}$ rotation in the opposite direction

if its value is small compared to the one of the signal, the window is declared as "non-activity" and the whole window signal is replaced by its mean (low pass filter that keeps only the constant component); (2) if not, the signal is kept as is (all-pass filter). This filter can be modelled as follows:

$$
\forall i \in\left[1 ; N_{w}\right], x_{i}= \begin{cases}x_{i} & \text { if } s_{w}>\epsilon \cdot s_{t} \\ \overline{x_{w}} & \text { else }\end{cases}
$$

with:

- $N_{w}$ The size of the Window

- $s_{w}$ Standard deviation of the Window

- $\epsilon$ a coefficient determined by experimentation

- $\overline{x_{w}}$ Mean value on the window

Results of the above-mentioned process are illustrated on Fig. 3-C and Fig. 4-C. They show that most of the windows are not processed due to their classification as "nonactivity" windows. This could allow to decrease time and calculus consumption, that could be highly relevant if we aim at developping embedded systems for tracking changes of directions of a person. Note that even if this filter creates a discontinuity in the signal (see Fig. 4-C), the processe is not impaired. Indeed, when a "non-activity" window is detected, the starting point of the analysis is automatically set at the first point of the next "activity" window.

\section{Movement clustering}

As we can see on Fig. 4-C, change of direction of a walking subject yields a significant increase in the euclidian 
norm of the difference between the two points in the plan $(\mathbf{x} ; \mathbf{y})$. Within this context, the changes in direction can be detected in the signal by a significant increased in the euclidian norm of the difference vector measured during two disctinct moments of the experiment. Let's define $\epsilon_{90}$ and $\epsilon_{180}$ as the two thresholds values corresponding to two rotation of $90^{\circ}$ and $180^{\circ}$, respectively. Interestingly, if the movement lasts more that 2 seconds, the analysis can be pursued on the next windows.

A change of direction of a walking person is detected when this euclidian norm is superior to $\epsilon_{90}$ and $\epsilon_{180}$ values. Furthermore, the direction of this change is indicated by the orientation of the difference vector.

On the whole, this filtering procedure, requiring to only compute euclidian norm of the three $\mathbf{x}, \mathbf{y}$ and $\mathbf{z}$ signals, is relatively rapid. However, this algorithm necessitates to verify the decision given by this filtering procedure by computing the real yaw angle of the signal. Indeed, one of the drawback concerns the correctness regarding to orientation of the movement. Indeed, outside the interval $\left[-\frac{\pi}{2} ; \frac{\pi}{2}\right]$, the classes for "plus" or "minus" angles are inverted. Once a false decision is taken, all the following orientations are false.

\section{Uncertainty minimisation}

1) Quaternion of the rotation: To check the last decision independantly of the previous ones, we compute the real yaw angle of the transformation by using the quaternion that describes the composition of the three rotations (around $\mathbf{x}, \mathbf{y}$ and $\mathbf{z}$ ). A preliminary step is to normalize the values delivered by the conditioning circuit positioned behind the magnetometers. Considering that (1) this conditioning circuit delivers linearly transformed values, and (2) each indivual has morphological differences, we center the data from the $(\mathbf{x}, \mathbf{y})$ plan in the interval $[-1 ; 1]$ for the subject, by using a first $360^{\circ}$ movement performed at the beginning of the experiment.

The norm of the magnetic field vector is almost constant (the small variations are caused by the acquisition noise) during the experimentation session. We measure the variable projection of a fixed vector on a moving referential (attached to the body of the subject).

To compute the yaw angle of the transformation, we first determine the quaternion of the transformation. Let's consider $\mathbf{B}_{1}$ and $\mathbf{B}_{2}$ the magnetic field measured at two moments of the experimentation. We want to determine a rotation of vector $\mathbf{k}=\left(\begin{array}{lll}k_{x} & k_{y} & k_{z}\end{array}\right)^{T}$ and of angle $\theta$ that transforms $\mathbf{B}_{\mathbf{1}}$ in $\mathbf{B}_{\mathbf{2}}$. Then, the quaternion is:

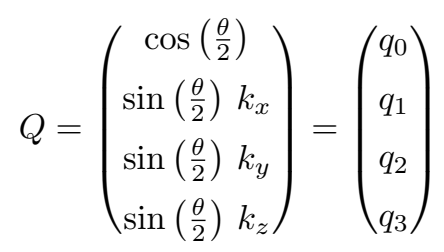

One possible basis in which the rotation can be written as
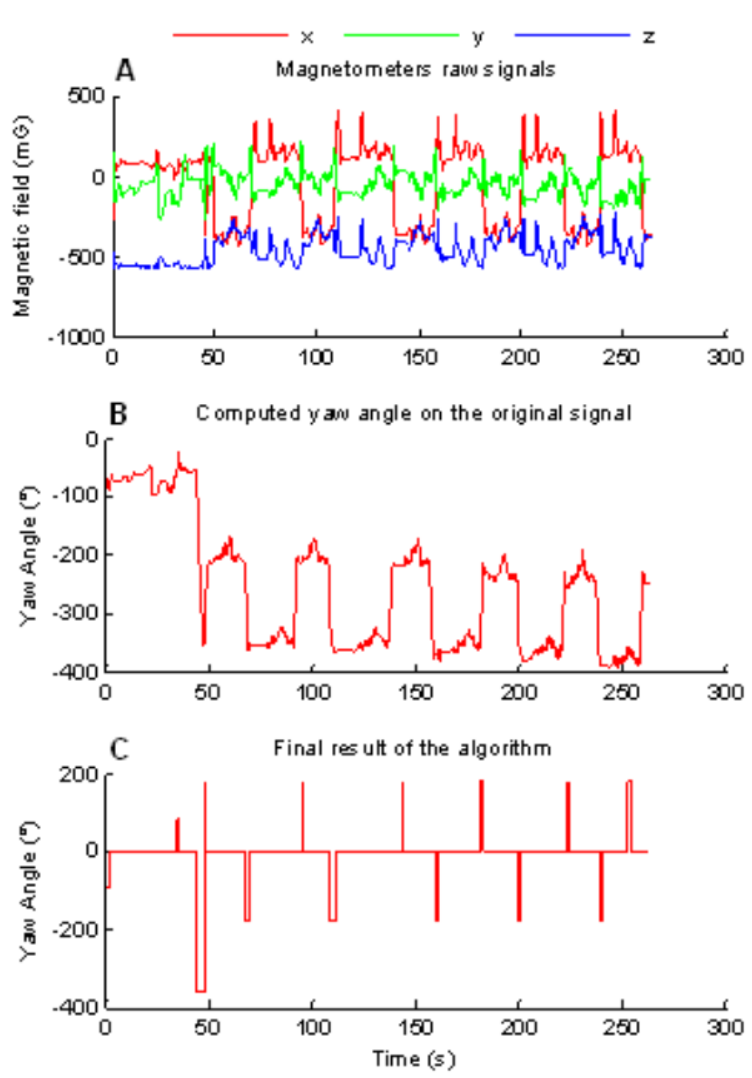

Fig. 5. Results of the execution of the algorithm on real data

a simple rotation of angle $\theta$ around one axis $\mathbf{k}$ is given by the set of three vectors:

$$
\left\{\begin{aligned}
\mathbf{i} & =\frac{\mathbf{B}_{\mathbf{1}}}{\left\|\mathbf{B}_{\mathbf{1}}\right\|} \\
\mathbf{j} & =\frac{\left\|\mathbf{B}_{\mathbf{1}}\right\|^{2} \mathbf{B}_{\mathbf{2}}-\left(\mathbf{B}_{1} \cdot \mathbf{B}_{2}\right) \mathbf{B}_{1}}{\|\| \mathbf{B}_{\mathbf{1}}\left\|^{2} \mathbf{B}_{2}-\left(\mathbf{B}_{1} \cdot \mathbf{B}_{2}\right) \mathbf{B}_{1}\right\|} \\
\mathbf{k} & =\frac{\mathbf{B}_{\mathbf{1}} \wedge \mathbf{B}_{\mathbf{2}}}{\left\|\mathbf{B}_{\mathbf{1}} \wedge \mathbf{B}_{\mathbf{2}}\right\|}
\end{aligned}\right.
$$

Let $\mathbf{b}_{1}$ be the projection of $\mathbf{B}_{1}$ in the basis given by Eq. 5 and $\mathbf{b}_{2}$ the projection of $\mathbf{B}_{2}$ in the same basis. The angle of the transformation is given by the expression:

$$
\theta=\arccos \left(\frac{\mathbf{b}_{\mathbf{1}} \cdot \mathbf{b}_{\mathbf{2}}}{\left\|\mathbf{b}_{\mathbf{1}}\right\| \cdot\left\|\mathbf{b}_{\mathbf{2}}\right\|}\right)
$$

With Eq. 5 and Eq. 6, we calculated the main vector and the angle of this rotation. We have now a numerical expression of the quaternion $Q$ given by Eq. 4 .

From this quaternion, we can write the rotation matrix in the basis $(\mathbf{x}, \mathbf{y}, \mathbf{z})$. The expression of this matrix $M_{r_{x y z}}$ is:

$$
\left(\begin{array}{ccc}
q_{0}^{2}+q_{1}^{2}-q_{2}^{2}-q_{3}^{2} & 2\left(-q_{0} q_{3}+q_{1} q_{2}\right) & 2\left(q_{0} q_{2}+q_{1} q_{3}\right) \\
2\left(q_{0} q_{3}+q_{2} q_{1}\right) & q_{0}^{2}-q_{1}^{2}-q_{2}^{2}-q_{3}^{2} & 2\left(-q_{0} q_{1}+q_{2} q_{3}\right) \\
2\left(-q_{0} q_{2}+q_{3} q_{1}\right) & 2\left(q_{0} q_{1}+q_{3} q_{2}\right) & q_{0}^{2}-q_{1}^{2}-q_{2}^{2}+q_{3}^{2}
\end{array}\right)
$$


2) Roll, pitch and yaw angle: Assuming that our movement is made of three rotations, one around the $\mathbf{z}$ axis (yaw), of angle $\psi$, the second of angle $\phi$ around $\mathbf{y}$, called pitch and the third, the roll angle, $\theta$, around $\mathbf{x}$, we compute the yaw angle of the rotation.

Let $M_{\psi}$ be the matrix of rotation around $\mathbf{z}, M_{\phi}$ around $\mathbf{y}$ and $M_{\theta}$ around $\mathbf{x}$. To compute the roll, pitch and yaw angles, we can identify the product of these three matrix, $M_{r_{x y z}}^{\prime}=M_{\psi} M_{\phi} M_{\theta}$, with the matrix given by Eq. 7 .

We obtain, with the same notations as previously :

$$
\left\{\begin{aligned}
\psi & =\arctan \left(\frac{M_{r_{x y z}}(1,2)}{M_{r_{x y z}}(1,1)}\right) \\
\theta & =\arcsin \left(M_{r_{x y z}}(1,3)\right) \\
\phi & =\arctan \left(\frac{M_{r_{x y z}}(2,3)}{M_{r_{x y z}}(3,3)}\right)
\end{aligned}\right.
$$

Since our application concerns the tracking of changes of direction of a person, only $\psi$ is of interest and will be computed for the period of time that we are currently checking only. To avoid any ambiguity, the computed yaw angle for the whole signal of Fig. 5-A is illustrated in Fig. 5-B.

\section{E. Final output of the algorithm}

After the euclidian norm having being computed and the decision having being verified by the quaternion, the executed movement has been detected accurately. This decision allows the calculation of final output of the algorithm, as follows. As illustrated in Fig. 5-C, for the temporal frame during which the movement takes place, the final output of the algorithm is the value corresponding to the angle induced by the executed rotation. this output gives us two main information: (1) the angle of the modification of direction and (2) the time spent to perform this rotation.

\section{RESULTS}

Eight young healthy adults (mean age : $27 \pm 3$ ) voluntarily participated to two experiments. Before these experiments, each subject stood upright and were asked to perform a $360^{\circ}$ rotation follwed by a $180^{\circ}$ rotation to calibrate the algorithm. In Experiment 1, they were asked to walk in a corridor, at a confortable speed, to make multiples changes of direction (ten $180^{\circ}$ changes, five in each direction of rotation) and to sit on a chair between these changes. In Experiment 2 , they were asked to walk, to go up and down the stairs and to make four $180^{\circ}$ changes (two in each direction) and eight $90^{\circ}$ changes (four in each direction). Results showed that our algorithm was able to accurately detect and classify changes of direction exectued by each subject during each experiment.

\section{Discussion AND CONCLUSION}

We have demonstrated the effectiveness of using a tri-axis magnetometer in accurately tracking the changes of directions of a person. Our algorithm, analysing the magnetometer signals by 2 seconds windows, is relatively rapid and can be executed in real time.

On the one hand, since the effectiveness of this algorithm mainly stem from the determination of the two values of $\epsilon_{90}$ and $\epsilon_{180}$, pursuing investigations to increase their optimality is necessary.

On the other hand, it is important to mention that our experiments were not conducted in "ideal" situations, i.e. without any magnetic disturbances. On the contrary, both experimental sessions required subjects to walk near TV, fridge, ... representing similar conditions to those occuring at home. What is more, we further assessed the electromagnetic sensitivity. To achieve this goal, we positioned the magnetometer $20 \mathrm{~cm}$ away from a TV for 30 minutes and we compared the norm of the magnetic field acquired and the standard deviation of this norm obtained when the TV was on to those obtained when the TV was off. Results showed similar magnetic field in these two conditions (mean measured magnetic field of $528.9 \mathrm{mG} \pm 1.94$ and of $528.7 m G \pm 1.65$, for TV off and TV on, respectively). Similar results were observed with fridge, microwave, fan and CRT display. On the whole, results showed that neither the TV, fridge, microwave, fan nor CRT display have any notable effect on the magnetometer acquisition circuit. Nevertheless, mean measured magnetic field obtained with the magnetometer positioned close to the elevator is significantly different to that obtained with the magnetometer positioned $1 \mathrm{~m}$ away from the elevator (2193.64 $m G \pm 303.13$ versus $565.19 m G \pm 10.21$ ). This result suggests that being very closed to an elevator, or more largely to a direct current engine, induces a large modification of the acquired magnetic field, that can be detected by our algorithm, but that limits the use of the magnetometers for tracking changes of direction of a person.

\section{ACKNOWLEDGMENT}

The authors would like to thanks Damien Flammarion and Jérémy Groussier for their help on the design of the sensors circuit and all the subjects from the TIMC-IMAG laboratory for their time accorded to these experimentations.

\section{REFERENCES}

[1] C. N. Scanaill, S. Carew, P. Barralon, N. Noury, D. Lyons, and G. M. Lyons, "A review of approaches to mobility telemonitoring of the elderly in their living environment," Annals of Biomedical Engineering, vol. 34, pp. 547 - 563, April 2006.

[2] A. K. Bourke, J. V. O'brien, and G. M. Lyons, "Evaluation of a threshold-based tri-axial accelerometer fall detection algorithm." Gait Posture, vol. 26, no. 2, pp. 194-199, Jul 2007.

[3] P. Barralon, N. Noury, and N. Vuillerme, "Classification of daily physical activities from a single kinematic sensor," in Engineering in Medicine and Biology Society, 2005. IEEE-EMBS 2005. 27th Annual International Conference of the, 2005, pp. 2447-2450.

[4] R. B. McGhee, E. R. Bachmann, X. Yun, and M. J. Zyda, "An investigation of alternative algorithms for singularity - free estimation of rigid body orientation from earth gravity and magnetic field measurements," Naval Postgraduate School, Monterey, California, Tech. Rep., 2001.

[5] R. Zhu and Z. Zhou, "A real-time articulated human motion tracking using tri-axis inertial/magnetic sensors package," IEEE Trans. Neural Syst. Rehab. Eng., vol. 12, no. 2, pp. 295 - 301, June 2004. 